\title{
First-principles calculations of the crystal structure, electronic structure, and thermodynamic stability of $\mathrm{Be}\left(\mathrm{BH}_{4}\right)_{2}$
}

\author{
Michiel J. van Setten and Gilles A. de Wijs \\ Electronic Structure of Materials, Institute for Molecules and Materials, Faculty of Science, Radboud University Nijmegen, \\ Toernooiveld 1, 6525 ED Nijmegen, The Netherlands \\ Geert Brocks \\ Computational Materials Science, Faculty of Science and Technology and MESA + Institute for Nanotechnology, University of Twente, \\ P.O. Box 217, 7500 AE Enschede, The Netherlands
}

(Received 6 December 2007; revised manuscript received 22 February 2008; published 10 April 2008)

\begin{abstract}
Alanates and boranates are intensively studied because of their potential use as hydrogen storage materials. In this paper, we present a first-principles study of the electronic structure and the energetics of beryllium boranate $\left[\mathrm{Be}\left(\mathrm{BH}_{4}\right)_{2}\right]$. From total energy calculations, we show that-in contrast to the other boranates and alanates-hydrogen desorption directly to the elements is likely and is at least competitive with desorption to the elemental hydride $\left(\mathrm{BeH}_{2}\right)$. The formation enthalpy of $\mathrm{Be}\left(\mathrm{BH}_{4}\right)_{2}$ is only $-0.14 \mathrm{eV} / \mathrm{H}_{2}$ (at $\left.T=0 \mathrm{~K}\right)$. This low value can be rationalized by the participation of all atoms in the covalent bonding, which is in contrast to the ionic bonding observed in other boranates. From calculations of thermodynamic properties at finite temperature, we estimate a decomposition temperature of $162 \mathrm{~K}$ at a pressure of $1 \mathrm{bar}$.
\end{abstract}

DOI: 10.1103/PhysRevB.77.165115

PACS number(s): 71.20.Dg, 61.50.Lt, 65.40.-b, 71.15.Mb

\section{INTRODUCTION}

In the past decade, the environmental importance of reducing the $\mathrm{CO}_{2}$ exhaust has been widely accepted. The use of hydrogen based fuel cells is an important contribution to achieve this reduction. One major obstacle for this use is the development of a method for hydrogen storage with a high gravimetric and volumetric hydrogen density. ${ }^{1}$

One way of storing hydrogen is in a (complex) metal hydride. The ideal hydrogen storage material should have the highest possible gravimetric hydrogen density. This obviously requires the use of lightweight materials. Moreover, the formation energy of the hydride has to be such that it is stable at atmospheric conditions; yet, it has to decompose at a moderate temperature to release the hydrogen. A further important point is that the reactions involved in hydrogen desorption and/or absorption must have fast kinetics.

Over the past decade, alanates and boranates have been extensively studied as potential hydrogen storage materials. ${ }^{1,2}$ Alanates and boranates consist of a lattice of metal cations and $\left(\mathrm{AlH}_{4}\right)^{-}$or $\left(\mathrm{BH}_{4}\right)^{-}$complex anions, respectively. Generally, these materials decompose by heating via intermediate complex hydrides into bulk metals, elemental hydrides, and hydrogen gas. In the past few years, the attention has gradually shifted from alanates toward boranates because of the high gravimetric hydrogen density in the latter. However, many boranates turn out to be too stable.

In principle, a large variety of boranates can be synthesized by changing the metal cations, which can be used to tune the formation energy. ${ }^{3}$ So far, most effort has been devoted to alkali boranates, ${ }^{4-10}$ and more recently, to mixtures of alkali boranates ${ }^{11,12}$ and to alkaline earth boranates. ${ }^{13-17} \mathrm{In}$ order to understand the chemical trends, we have recently developed a simple model for the formation energies of these compounds. ${ }^{18}$ This model demonstrates that these boranates are ionic compounds (in the sense discussed above) and that the difference in their formation energies can be understood on the basis of the electrostatic (Madelung) lattice energy. The basic stability of the $\left(\mathrm{BH}_{4}\right)^{-}$cation is not affected by substituting one alkali or alkaline earth cation by another.

The stability of $\left(\mathrm{BH}_{4}\right)^{-}$may be changed by adding an element that competes with boron in binding with hydrogen. To investigate this possibility, we study beryllium boranate ${ }^{19}$ $\left[\mathrm{Be}\left(\mathrm{BH}_{4}\right)_{2}\right]$ in this paper. Establishing the electronic structure and thermodynamic stability of $\mathrm{Be}\left(\mathrm{BH}_{4}\right)_{2}$ will assist us in understanding the chemical and physical trends in alkali, alkaline earth alanates, and boranates. ${ }^{20}$

We present a first-principles study of the electronic structure and the thermodynamic properties of $\mathrm{Be}\left(\mathrm{BH}_{4}\right)_{2}$. The electronic structure in relation to the crystal structure is used to analyze the bonding in $\mathrm{Be}\left(\mathrm{BH}_{4}\right)_{2}$. We calculate total energies and phonon frequencies of all compounds involved in possible formation reactions of $\mathrm{Be}\left(\mathrm{BH}_{4}\right)_{2}$. From these data, we obtain the thermodynamic properties at finite temperature.

\section{COMPUTATIONAL METHODS}

First-principles calculations are carried out within the density functional theory (DFT) approach by applying a generalized gradient approximation (GGA) for the exchange correlation functional. ${ }^{21}$ We use a plane wave basis set and the projector augmented wave (PAW) method, ${ }^{22,23}$ as implemented in the Vienna ab initio simulation package (VASP), ${ }^{24-26}$ and apply nonlinear core corrections. ${ }^{27}$

Brillouin zone integrations are performed with a tetrahedron method ${ }^{28}$ for calculating total energies. A Gaussian smearing method is used for calculating densities of states, with a smearing parameter of $0.1 \mathrm{eV}$. The $k$-point meshes are such that total energies are converged within $0.1 \mathrm{meV} / \mathrm{f}$.u. The total energies used in the calculations of the reaction enthalpies are obtained with a high plane wave kinetic en- 


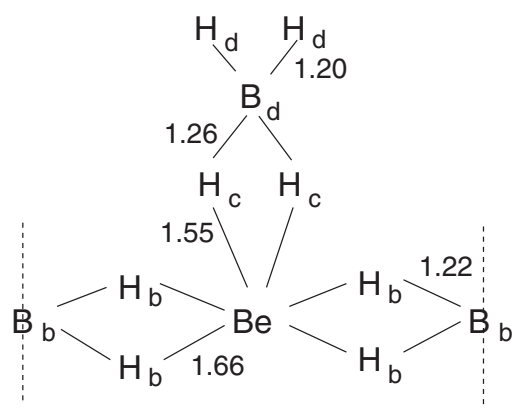

FIG. 1. Schematic bonding scheme and labeling of the atoms within a polymer chain in $\mathrm{Be}\left(\mathrm{BH}_{4}\right)_{2}$. The three dimensional structure is given in Fig. 2. The numbers indicate optimized bond lengths in angstrom.

ergy cutoff of $700 \mathrm{eV}$. By varying the computational parameters, in particular, by trying different PAW potentials, ${ }^{29}$ we estimate that reaction enthalpies are converged on a scale of $5 \mathrm{meV}$. For the cohesive energy of $\mathrm{Be}$ and the formation energy of $\mathrm{BeH}_{2}$ (see Sec. V), we obtain results very similar to the DFT results of Hector et al. ${ }^{30}$

The atomic positions and lattice parameters are relaxed using a conjugate gradient algorithm for a range of fixed volumes. The total energy versus volume curve obtained this way is fitted with Murnaghan's equation of state expression, ${ }^{31}$ which yields the ground state volume, the bulk modulus, and its pressure derivative. At the ground state volume, we relaxed the atomic positions and lattice parameters to obtain the ground state structure. This procedure is followed for all compounds mentioned in this paper.

To calculate the zero point energies (ZPEs) and phonon densities of state, we need the phonon frequencies of all of these compounds. Vibrational frequencies are obtained from the dynamical matrix, whose matrix elements (the force constants) are calculated using a finite difference method. ${ }^{32}$ The force constants are calculated from displacements of $0.005 \AA$ in two opposite directions for each atomic degree of freedom. For both bulk beryllium and beryllium hydride, $2 \times 2$ $\times 2$ supercells give converged ZPEs. One does not need a supercell to calculate the phonon frequencies of $\mathrm{Be}\left(\mathrm{BH}_{4}\right)_{2}$ since the unit cell of $\mathrm{Be}\left(\mathrm{BH}_{4}\right)_{2}$ is sufficiently large. For boron, we use the frequencies that have been reported earlier. ${ }^{33}$

\section{CRYSTAL STRUCTURE}

$\mathrm{Be}\left(\mathrm{BH}_{4}\right)_{2}$ can be synthesized by the reaction of lithium boranate and beryllium chloride. ${ }^{34,35}$ Its crystal structure consists of helical polymers of alternating beryllium and boron atoms $\left(\mathrm{B}_{b}\right)$ that are connected via pairs of hydrogen atoms $\left(\mathrm{H}_{b}\right){ }^{36}$ The polymer building block is schematically shown in Fig. 1. A further boron atom $\left(\mathrm{B}_{d}\right)$ is attached to each beryllium atom, again via a pair of hydrogen atoms $\left(\mathrm{H}_{c}\right)$, and this $\mathrm{B}_{d}$ atom also binds two "dangling" hydrogen atoms $\left(\mathrm{H}_{d}\right)$. The polymers are packed in the crystal structure, as shown in Fig. 2. On the basis of this structure, one may expect a strong bonding between the atoms in one polymer chain and a much weaker bonding between the polymer

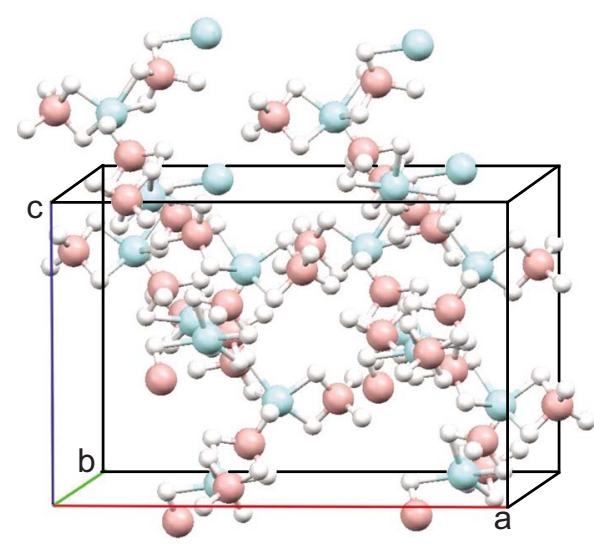

FIG. 2. (Color online) Crystal structure of $\mathrm{Be}\left(\mathrm{BH}_{4}\right)_{2}$. Hydrogen atoms (small white spheres) form tetrahedra around the boron atoms [pink (dark gray) spheres]. Each beryllium atom [blue (light gray) spheres] is bonded to three $\mathrm{BH}_{4}$ tetrahedra.

chains. The latter is reflected in the low melting point of $\mathrm{Be}\left(\mathrm{BH}_{4}\right)_{2}$ of $125^{\circ} \mathrm{C}$.

We relaxed the crystal structure of $\mathrm{Be}\left(\mathrm{BH}_{4}\right)_{2}$, as described in the previous section, including the cell volume, lattice parameters, and atomic positions, while keeping the experimental space group and Wyckoff positions. The optimized lattice parameters and atomic positions are given in Table I. The calculated lattice parameters are 5\% larger than the experimental values..$^{36}$ This is consistent with the weak binding between the polymer chains, which is of van der Waals type. It is well known that, using the common functionals, DFT fails to capture van der Waals bonding and overestimates cell parameters and volumes in weakly bonded systems. However, the total energy difference between the experimental and calculated cell volumes is less than $5 \mathrm{meV} / \mathrm{H}_{2}$ (see Fig. 3 ). This error only has a minor effect on the relative total energies.

TABLE I. Optimized atomic positions of $\mathrm{Be}\left(\mathrm{BH}_{4}\right)_{2}$. The space group is $I 4_{1} c d$ (No. 110) and all atoms are on $16 b$ Wyckoff positions. The optimized lattice parameters are $a=b=14.28 \AA$ and $c$ $=9.54 \AA$ (the cell volume is $1943.90 \AA^{3}$ ). The experimental values are $a=b=13.62 \AA$ and $c=9.10 \AA$ (cell volume is $1688.09 \AA^{3}$ ) (Ref. 36).

\begin{tabular}{lccc}
\hline \hline Atom & $x$ & $y$ & $z$ \\
\hline $\mathrm{Be}$ & 0.2050 & 0.0992 & 0.0016 \\
$\mathrm{~B}_{d}$ & 0.1695 & 0.9702 & 0.0068 \\
$\mathrm{~B}_{b}$ & 0.1503 & 0.1978 & 0.1237 \\
$\mathrm{H}_{d}$ & 0.0997 & 0.9439 & 0.0653 \\
$\mathrm{H}_{d}$ & 0.2183 & 0.9130 & 0.9499 \\
$\mathrm{H}_{c}$ & 0.2189 & 0.0123 & 0.0963 \\
$\mathrm{H}_{c}$ & 0.1453 & 0.0294 & 0.9157 \\
$\mathrm{H}_{b}$ & 0.1083 & 0.1649 & 0.0231 \\
$\mathrm{H}_{b}$ & 0.2281 & 0.1647 & 0.1450 \\
$\mathrm{H}_{b}$ & 0.1611 & 0.2813 & 0.1003 \\
$\mathrm{H}_{b}$ & 0.1027 & 0.1793 & 0.2269 \\
\hline \hline
\end{tabular}




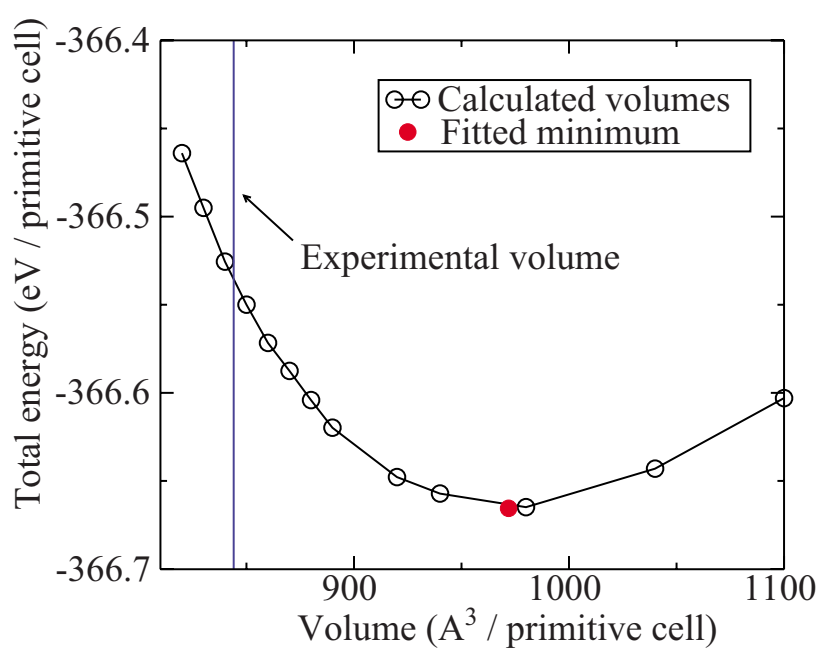

FIG. 3. (Color online) Total energy per unit cell of $\mathrm{Be}\left(\mathrm{BH}_{4}\right)_{2}$ as a function of the cell volume.

The optimized $\mathrm{B}-\mathrm{H}$ and $\mathrm{Be}-\mathrm{H}$ bond lengths are given in Fig. 1. As references, the $\mathrm{B}-\mathrm{H}$ bond length in a $\left(\mathrm{BH}_{4}\right)^{-}$anion is $1.21 \AA$, whereas a $\mathrm{B}-\mathrm{H}$ bond length in a typical three center $\mathrm{B}-\mathrm{H}-\mathrm{B}$ bond is $1.34 \AA{ }^{34} \mathrm{~A}$ comparison with these numbers indicates that the $\mathrm{B}-\mathrm{H}$ bonding in $\mathrm{Be}\left(\mathrm{BH}_{4}\right)_{2}$ is closer to that in the $\left(\mathrm{BH}_{4}\right)^{-}$, although there is some distortion due to the presence of the $\mathrm{Be}$ atom, in particular, on the $\mathrm{B}_{d}-\mathrm{H}_{c}$ bond. This could indicate some competition between $\mathrm{B}$ and $\mathrm{Be}$ for bonding to hydrogen. For comparison, the B-H bond lengths in alkali boranates are all very close to $1.21 \AA$. However, the Be-H bond lengths are still quite large, which indicates a significant ionic contribution to the bonding.

We have also optimized the structure of $\mathrm{BeH}_{2}$ (see Table II). It agrees well with the experimental structure ${ }^{37}$ and with that obtained in a previous calculation, ${ }^{30,38}$ the largest difference being that our calculated bulk modulus $(21.4 \mathrm{GPa})$ is $\sim 10 \%$ smaller than that calculated in Ref. 38 (23.8 GPa). For elemental boron, we use the $\beta$-rhombohedral structure, as given in Ref. 33. For elemental beryllium [space group $P \overline{3} m 1$ (No. 164)], we find lattice parameters $a=2.260 \AA$ and $c=3.567 \AA$, which compare well with the experimental values of 2.29 and $3.60 \AA$, respectively. ${ }^{39}$

\section{ELECTRONIC STRUCTURE}

As discussed above, the crystal structure of $\mathrm{Be}\left(\mathrm{BH}_{4}\right)_{2}$ indicates a weak bonding between polymer chains and a stron-

TABLE II. Optimized crystal structure of $\mathrm{BeH}_{2}$. The space group is Ibam (No. 72) and the optimized lattice parameters are $a, b, c=8.967,4.141,7.643 \AA$. The experimental lattice parameters are $a, b, c=9.082,4.160,7.707 \AA$ (Ref. 37).

\begin{tabular}{lclll}
\hline \hline Atom & Wycoff & \multicolumn{1}{c}{$x$} & \multicolumn{1}{c}{$y$} & \multicolumn{1}{c}{$z$} \\
\hline $\mathrm{Be}$ & $4 a$ & 0 & 0 & 0.25 \\
$\mathrm{Be}$ & $8 j$ & 0.1677 & 0.1200 & 0 \\
$\mathrm{H}$ & $16 k$ & 0.0882 & 0.2241 & 0.1520 \\
$\mathrm{H}$ & $8 j$ & 0.3102 & 0.2771 & 0 \\
\hline \hline
\end{tabular}

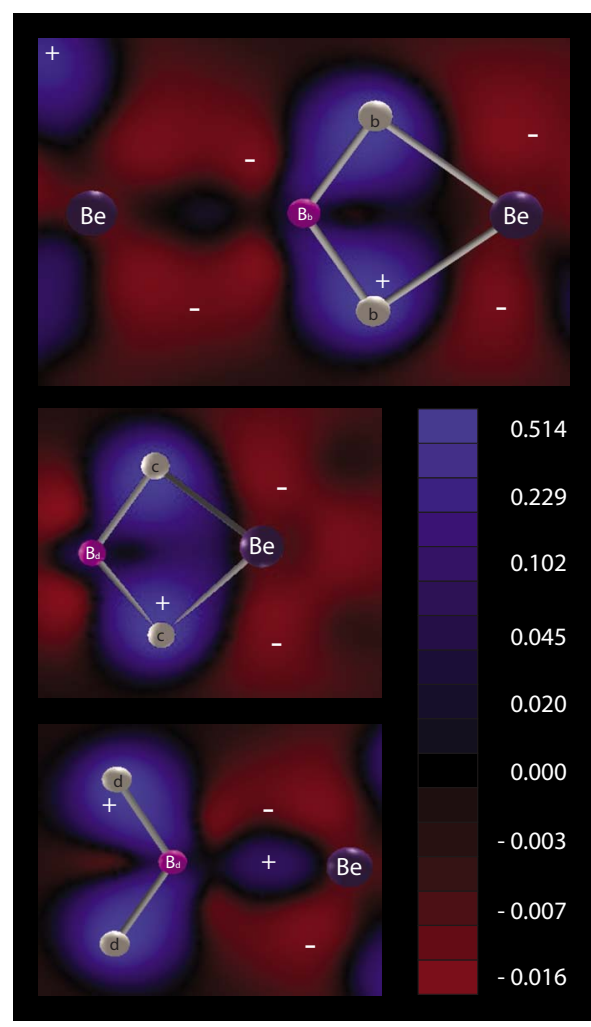

FIG. 4. (Color online) Charge density difference plots $\left(e / \AA^{3}\right)$ of $\mathrm{Be}\left(\mathrm{BH}_{4}\right)_{2}$ with respect to the isolated atoms. The top picture gives a cut through a plane containing $\mathrm{B}_{b}, \mathrm{H}_{b}$, and $\mathrm{Be}$ atoms of the polymer backbone, the middle picture a cut through $\mathrm{B}_{d}$ and $\mathrm{H}_{c}$ side chain plane, and the bottom right picture a cut through $\mathrm{B}_{d}$ and $\mathrm{H}_{d}$ side chain plane. Electrons are transferred from regions close to the $\mathrm{Be}$ atoms to regions close to the $\mathrm{H}$ atoms and the $\mathrm{B}-\mathrm{H}$ bonds.

ger bonding within a polymer chain. The charge displacement upon bond formation can be visualized by plotting the charge density difference, i.e., the charge density of $\mathrm{Be}\left(\mathrm{BH}_{4}\right)_{2}$ minus that of the individual isolated atoms. Cuts through the charge density difference in various planes along a polymer backbone are shown in Fig. 4. They clearly indicate the formation of B-H covalent bonds, which are polarized somewhat toward the $\mathrm{H}$ atoms. The character of the $\mathrm{Be}-\mathrm{H}$ bonds is much less clear from these plots. In any case, these bonds are strongly polarized in the direction of the $\mathrm{H}$ atoms.

The electronic projected density of states (PDOS) of $\mathrm{Be}\left(\mathrm{BH}_{4}\right)_{2}$, with projections on $s, p$ components of the individual atoms, is shown in Fig. 5. Tetrahedrally bonded $\left(\mathrm{BH}_{4}\right)^{-}$generates a characteristic pattern in the valence band part of the PDOS, which is qualitatively similar to that observed for $\left(\mathrm{AlH}_{4}\right)^{-}$tetrahedra in the alanates. ${ }^{40-45}$ The tetrahedral geometry of $\left(\mathrm{BH}_{4}\right)^{-}$results in a splitting into two valence peaks, i.e., the lower one of $s\left(A_{1}\right)$ symmetry and the upper one of $p\left(T_{2}\right)$ symmetry, with a relative weight ratio of 1:3. Projected on atomic states, the $s$ peak then has contributions from $\mathrm{H} s$ and B $s$ orbitals and the $p$ peak has contributions from $\mathrm{H} s$ and B $p$ orbitals. The $p$ peak can be split due to symmetry breaking caused by the crystal field. This is clearly observed in the lowest two panels of Fig. 5, showing 


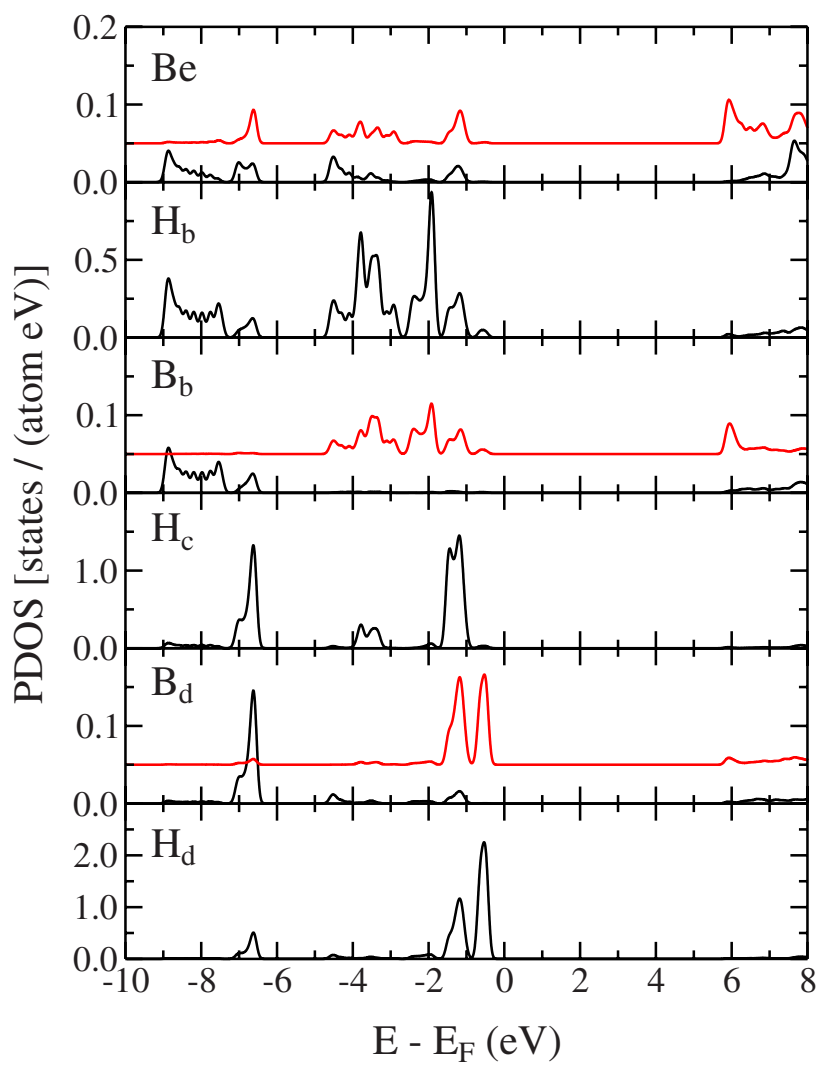

FIG. 5. (Color online) The PDOS of $\mathrm{Be}\left(\mathrm{BH}_{4}\right)_{2}$. The Fermi level $E_{F}$ at the top of the valence band is the zero of energy. The upper (red) and lower curves (black) give projections on $p$ and $s$ atomic states, respectively. Atomic radii of $0.7,0.5$, and $1.1 \AA$ are used for $\mathrm{Be}, \mathrm{B}$, and $\mathrm{H}$, respectively.

the PDOS on the $\mathrm{B}_{d}$ and $\mathrm{H}_{d}$ atoms with the $s$ peak at $\sim-7 \mathrm{eV}$ and a $p$ doublet around $\sim-1 \mathrm{eV}$. The splitting between $s$ and $p$ peaks is large $(\sim 6 \mathrm{eV})$, and the crystal field splitting is much smaller $(\sim 1 \mathrm{eV})$.

The interaction between the $\mathrm{BH}_{4}$ units in the crystal lattice leads to a broadening of the peaks due to band formation. The interaction is strongest along the $\mathrm{B}_{b}\left(\mathrm{H}_{b}\right)_{4}-\mathrm{Be}$ $-\mathrm{B}_{b}\left(\mathrm{H}_{b}\right)_{4}$ polymer backbone (see Figs. 1 and 2). This leads to an $s$-type band in the range of $\sim-9$ to $\sim-7.5 \mathrm{eV}$, involving contributions from $\mathrm{H}_{b}, \mathrm{~B}_{b}$, and Be $s$ orbitals, whose DOS has the characteristic shape of a one-dimensional structure (see the upper three panels of Fig. 5). In the range of $\sim-5$ to $\sim-2 \mathrm{eV}$, we find a set of $p$-type bands. The bandwidths are smaller than the $s p$ splitting, but they are not negligible, reflecting the covalent bonding along the polymer backbone.

The involvement of the Be atoms can be clarified by calculating the DOS for a $\mathrm{Be}\left(\mathrm{BH}_{4}\right)_{2}$ structure, in which the Be atoms are replaced by a homogeneous background with a charge of $2+$. The result is shown in Fig. 6. The $s$ and $p$ valence bands discussed above are replaced by much narrower peaks that reflect electron localization on $\left(\mathrm{BH}_{4}\right)^{-}$ions in this artificial structure. In other words, the Be atoms in $\mathrm{Be}\left(\mathrm{BH}_{4}\right)_{2}$ are involved in the covalent bonding. This is in contrast to alkali or alkaline earth boranates and alanates, where the DOS changes little if the cations are replaced by a background charge. The bonding in the latter compounds can

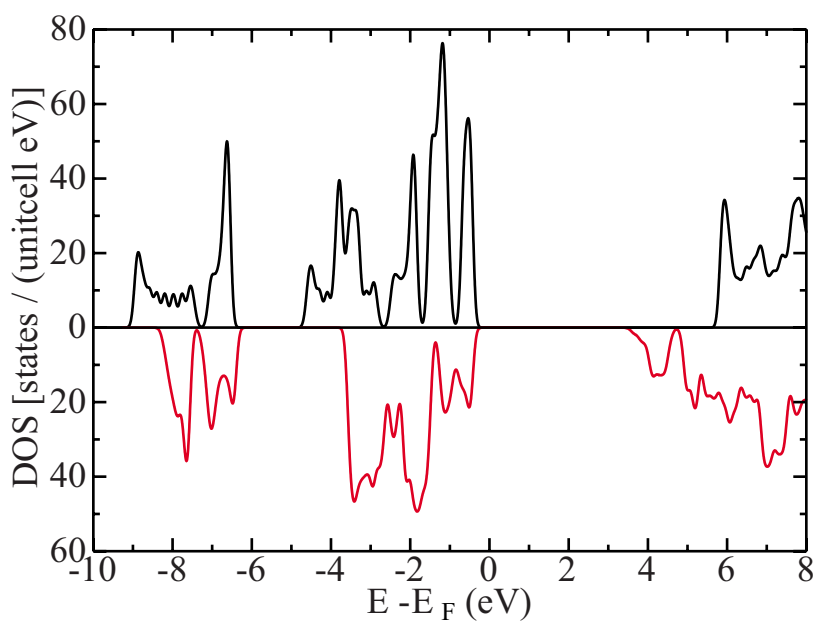

FIG. 6. (Color online) The total DOS of $\mathrm{Be}\left(\mathrm{BH}_{4}\right)_{2}$. The Fermi level $E_{F}$ at the top of the valence band is the zero of energy. In the lower curve, the beryllium atoms are replaced by an homogeneous background with a charge of $2+$.

be described as an ionic bonding between $\left(\mathrm{BH}_{4}\right)^{-}$or $\left(\mathrm{AlH}_{4}\right)^{-}$ anions and $M^{+}$(akali) or $M^{\prime 2+}$ (alkaline earth) cations. ${ }^{45}$

\section{REACTION ENTHALPIES}

For light elements, such as hydrogen, beryllium, and boron, the quantum character of their atomic vibrations is important. This leads to vibrational energies at zero temperature that are not negligible. For each compound involved in the reaction, we calculate its zero point vibrational energy (ZPVE) from the frequencies of the vibrational modes in the optimized structure. For hydrogen molecules, the zero point rotational energy (ZPRE) is also not entirely negligible. Reaction enthalpies $\Delta H_{0}$ at $T=0 \mathrm{~K}$ are then calculated from

$$
\Delta H_{0}=\sum_{p}\left(E_{p}^{\mathrm{tot}}+E_{p}^{\mathrm{ZPVE}}\right)+E_{H_{2}}^{\mathrm{ZPRE}}-\sum_{r}\left(E_{r}^{\mathrm{tot}}+E_{r}^{\mathrm{ZPVE}}\right),
$$

where $E_{p / r}^{\mathrm{tot}}$ denotes the total energy of the reaction products $p$ or reactants $r, E_{p / r}^{\mathrm{ZPVE}}$ are the corresponding ZPVEs, and $E_{H_{2}}^{\mathrm{ZPRE}}$ is the ZPRE of the hydrogen molecules involved in the reaction. At $T=0 \mathrm{~K}$, reaction enthalpies with neglected ZPEs will be denoted by $\Delta E$.

For the hydrogen molecules, we calculate a vibrational frequency of $4356 \mathrm{~cm}^{-1}$, which is in good agreement with the experimental value of $4401 \mathrm{~cm}^{-1} .46$ The ZPVE, i.e., $0.266 \mathrm{eV}$, is then calculated from the energy levels of a Morse potential,

$$
E(n)=\hbar \omega\left(n+\frac{1}{2}\right)-\frac{1}{4 D_{e}}\left[\hbar \omega\left(n+\frac{1}{2}\right)\right]^{2},
$$

where $\omega$ is the vibration eigenfrequency and $D_{e}=4.57 \mathrm{eV}$ is the dissociation energy. Assuming that ortho- and parahydrogen molecules are produced in a proportion of $3: 1$, the average ZPRE of a hydrogen molecule is $0.011 \mathrm{eV}$ by using the energy levels given in Ref. 46.

The calculated total energies and ZPEs of all compounds involved in the reactions are listed in Table III. We consider 
TABLE III. Total energies (with respect to nonspin polarized model atoms), ZPVEs, and ZPREs in eV/f.u. in the relaxed structures.

\begin{tabular}{lcll}
\hline \hline & $E^{\text {tot }}$ & $E^{\text {ZPVE }}$ & $E^{\text {ZPRE }}$ \\
\hline $\mathrm{Be}\left(\mathrm{BH}_{4}\right)_{2}$ & -45.868 & 2.450 & \\
$\mathrm{BeH}_{2}$ & -10.797 & 0.542 & \\
$\mathrm{H}_{2}$ & -6.803 & 0.266 & 0.011 \\
$\mathrm{~B}$ & -6.687 & 0.126 & \\
$\mathrm{Be}$ & -3.729 & 0.091 & \\
\hline \hline
\end{tabular}

two possible reaction paths for the formation of $\mathrm{Be}\left(\mathrm{BH}_{4}\right)_{2}$. In the first path, $\mathrm{Be}\left(\mathrm{BH}_{4}\right)_{2}$ is directly formed from the elements, as follows:

$$
\mathrm{Be}+2 \mathrm{~B}+4 \mathrm{H}_{2}(g) \rightarrow \mathrm{Be}\left(\mathrm{BH}_{4}\right)_{2} .
$$

The second path involves the formation of an intermediate compound $\mathrm{BeH}_{2}$, as follows:

$$
\begin{gathered}
\mathrm{Be}+\mathrm{H}_{2}(g) \rightarrow \mathrm{BeH}_{2}, \\
\mathrm{BeH}_{2}+2 \mathrm{~B}+3 \mathrm{H}_{2}(g) \rightarrow \mathrm{Be}\left(\mathrm{BH}_{4}\right)_{2} .
\end{gathered}
$$

The enthalpies of these reactions are calculated using Eq. (1) and the values are given in Table III.

Equation (3) gives a reaction enthalpy $\Delta E$ $=-0.39 \mathrm{eV} / \mathrm{H}_{2}$ if ZPEs are neglected. If ZPEs are included, the reaction enthalpy becomes $\Delta H_{0}=-0.14 \mathrm{eV} / \mathrm{H}_{2}$, which indicates the importance of ZPE corrections for these lightweight compounds. In principle, these values are in a range that is useful for hydrogen storage.

Can these enthalpies be understood as an extrapolation of reaction enthalpies of similar boranate systems? Recently, Nakamori et $a .^{3}$ observed a linear dependence of the (calculated) formation enthalpies of boranates $M\left(\mathrm{BH}_{4}\right)_{n}$ on the Pauling electronegativity $\chi_{P} S$ of the cation $M$. Smaller $\chi_{P} S$ correspond to more stable boranates. Using their linear fit and $\chi_{P}=1.57$ for $\mathrm{Be}$, we obtain $\Delta H_{0}=0.00 \mathrm{eV} / \mathrm{H}_{2}$. The deviation between this and our first-principles number $\left(-0.14 \mathrm{eV} / \mathrm{H}_{2}\right)$ is somewhat larger than the mean square deviation of $0.05 \mathrm{eV} / \mathrm{H}_{2}$ that Nakamori et al. ${ }^{3}$ obtained for their fit. In another recent study, we employed an ionic model with a modified Born-Haber cycle to analyze the formation energies of alanates and boranates. ${ }^{18}$ This model gives a reaction enthalpy $\Delta E=-0.02 \mathrm{eV} / \mathrm{H}_{2}$. This number is also higher than the value we obtain from first-principles calculation $\left(-0.39 \mathrm{eV} / \mathrm{H}_{2}\right)$. Both the analyses of Nakamori et al. ${ }^{3}$ and van Setten et al. ${ }^{18}$ were based on an ionic picture. However, in the previous section, we already concluded that the bonding in $\mathrm{Be}\left(\mathrm{BH}_{4}\right)_{2}$ is not purely ionic. The $\mathrm{Be}$ atoms are (partially) covalently bonded to $\mathrm{BH}_{4}$, which gives an additional stabilization, resulting in a higher dehydrogenation enthalpy.

Most alanates and boranates form a simple alkali and/or alkaline earth hydride when hydrogen is released in a first step. The dehydrogenation of this simple hydride then occurs as a separate second step. Usually, the enthalpies are such that only the first step is considered useful for hydrogen stor-
TABLE IV. Calculated reaction enthalpies at $0 \mathrm{~K}$ without ZPVE $(\Delta E)$, at $0 \mathrm{~K}$ including $\mathrm{ZPVE}\left(\Delta H_{0}\right)$, and at $298 \mathrm{~K}\left(\Delta H_{298}\right)$. All entries are in $\mathrm{eV} / \mathrm{H}_{2}(\mathbf{g})$.

\begin{tabular}{lccc}
\hline \hline & $\Delta E$ & $\Delta H_{0}$ & $\Delta H_{298}$ \\
\hline $\mathrm{Be}+2 \mathrm{~B}+4 \mathrm{H}_{2}(g) \rightarrow \mathrm{Be}\left(\mathrm{BH}_{4}\right)_{2}$ & -0.39 & -0.14 & -0.21 \\
$\mathrm{Be}+\mathrm{H}_{2}(g) \rightarrow \mathrm{BeH}_{2}$ & -0.27 & -0.09 & -0.16 \\
$\mathrm{BeH}_{2}+2 \mathrm{~B}+3 \mathrm{H}_{2}(g) \rightarrow \mathrm{Be}\left(\mathrm{BH}_{4}\right)_{2}$ & -0.43 & -0.15 & -0.22 \\
\hline \hline
\end{tabular}

age. For $\mathrm{Be}\left(\mathrm{BH}_{4}\right)_{2}$, these two steps correspond to the reverse reactions of Eqs. (5) and (4). The calculated reaction enthalpies of Eqs. (4) and (5) are $\Delta E=-0.27$ and $-0.43 \mathrm{eV} / \mathrm{H}_{2}(g)$ and $\Delta H_{0}=-0.09$ and $-0.15 \mathrm{eV} / \mathrm{H}_{2}(g)$ with $\mathrm{ZPE}$ corrections, respectively. By comparing these numbers to those of reaction (3), we see that (per $\left.\mathrm{H}_{2}\right) \mathrm{Be}\left(\mathrm{BH}_{4}\right)_{2}$ is slightly more stable than $\mathrm{BeH}_{2}$. This would indicate that a one-step reaction directly from the elements [Eq. (3)] is more favorable than the two-step reaction via the simple hydride [Eqs. (4) and (5)]. However, the enthalpy difference is very small. In addition, kinetic barriers may influence the relative importance of the two reaction paths.

We will now focus on finite temperature properties. For solids, we calculate the Gibbs free energy $G(T)$ in the harmonic approximation as

$$
G(T)=E^{\mathrm{tot}}+H^{\mathrm{vib}}(T)-T S^{\mathrm{vib}}(T),
$$

with

$$
H^{\mathrm{vib}}(T)=\int_{0}^{\infty} d \omega g(\omega)\left\{\frac{1}{2} \hbar \omega+\hbar \omega n(\omega)\right\}
$$

and

$$
S^{\mathrm{vib}}(T)=k_{B} \int_{0}^{\infty} d \omega g(\omega)\left\{\beta \hbar \omega n(\omega)-\ln \left[1-e^{-\beta \hbar \omega}\right]\right\},
$$

where $g(\omega)$ is the phonon density of states, $n(\omega)$ $=[\exp (\beta \hbar \omega)-1]^{-1}$ is the Bose-Einstein occupation number, and $\beta=1 / k_{B} T$. The first term in the integral of Eq. (7) gives the ZPVE and the second term gives the finite temperature contribution. Note that we neglect the $P V$ term (i.e., the distinction between energy and enthalpy), which is a good approximation for solids. For the Gibbs free energy, the enthalpy, and the entropy of the hydrogen gas, we use the values given in Ref. 47.

By using the above expressions, we obtain the enthalpies at $298 \mathrm{~K}$ and 1 bar. All enthalpies are summarized in Table IV. The calculated reaction enthalpy $\Delta H_{298}=-0.16 \mathrm{eV} / \mathrm{H}_{2}$ of Eq. (4) is in good agreement with $\Delta H_{298}=-0.15 \mathrm{eV} / \mathrm{H}_{2}$, which was obtained by Hector et al. ${ }^{30}$ in a similar DFT study. It is also in reasonable agreement with the experiment (for amorphous $\mathrm{BeH}_{2}$ ) of Senin et al., ${ }^{48}$ who obtained $\Delta H_{298}=-0.20 \mathrm{eV} / \mathrm{H}_{2}$. (For deuteride, the formation enthalpy for the crystalline state is available: $\Delta H_{298}\left(\mathrm{BeD}_{2}\right)$ $=-0.32 \mathrm{eV} / \mathrm{H}_{2}{ }^{48}$ By combining our $\Delta E$ with the temperature corrections from Ref. 30, we find $\Delta H_{298}\left(\mathrm{BeD}_{2}\right)$ $=-0.20 \mathrm{eV} / \mathrm{H}_{2}$, which is very close to the result of Ref. 30.) 


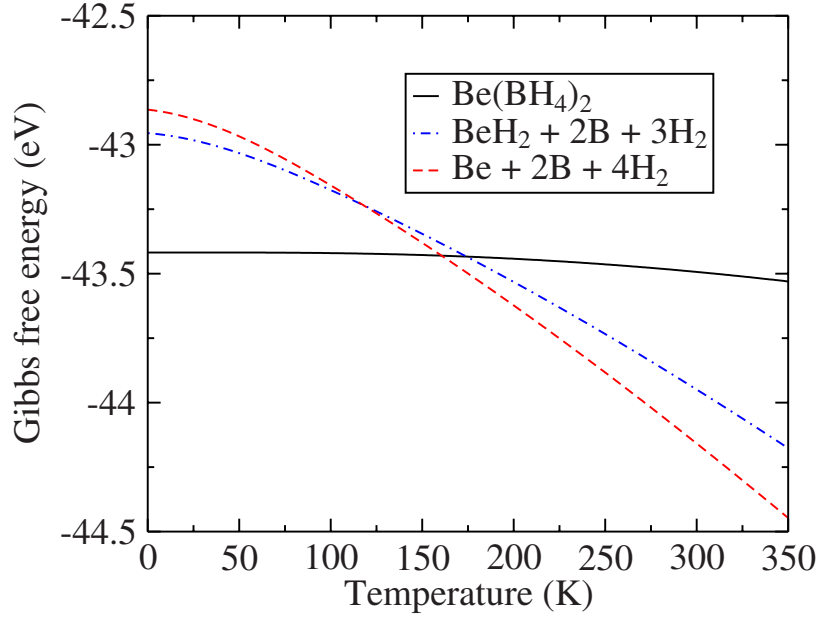

FIG. 7. (Color online) Gibbs free energy of $\mathrm{Be}\left(\mathrm{BH}_{4}\right)_{2}$ (solid line), $\mathrm{Be}+2 \mathrm{~B}+4 \mathrm{H}_{2}(g)$ (dashed red line), and $\mathrm{BeH}_{2}+2 \mathrm{~B}+3 \mathrm{H}_{2}(g)$ (dash dot blue line). All lines pertain to the same net composition of 1 f.u. $\mathrm{Be}\left(\mathrm{BH}_{4}\right)_{2}$.

Figure 7 gives the free energies $G(T)$ of $\mathrm{Be}\left(\mathrm{BH}_{4}\right)_{2}$, the products of the dehydrogenation reaction [i.e., the left and right hand sides of Eq. (3)], and the possible intermediate with $\mathrm{BeH}_{2}$ [the left hand side of Eq. (5)] at a standard pressure of 1 bar. At $162 \mathrm{~K}$, the free energy of the products drops below that of the hydride phase, whereas the "intermediate" with $\mathrm{BeH}_{2}$ remains above the most stable curve throughout most of the temperature range. It therefore seems unlikely that hydrogen desorption goes via an intermediate stage with $\mathrm{BeH}_{2}$. However, as previously noted above for the enthalpies, the Gibbs free energy curves come very close. Moreover, kinetic effects might play a role.

The predicted desorption temperature relies only on the thermodynamics of the reaction. From the fact that experimentally $\mathrm{Be}\left(\mathrm{BH}_{4}\right)_{2}$ seems to be stable at room temperature, one may conclude that kinetic barriers play an important role in stabilizing $\mathrm{Be}\left(\mathrm{BH}_{4}\right)_{2}$. In this respect, $\mathrm{Be}\left(\mathrm{BH}_{4}\right)_{2}$ is similar to other boranates and alanates, where decomposition temperatures are much higher than what is expected on the basis of thermodynamics, and catalysts have to be applied in order to overcome kinetic barriers.

\section{CONCLUSIONS}

We use DFT electronic structure calculations at the GGA level to study the crystal structure, electronic structure, and thermodynamics of $\mathrm{Be}\left(\mathrm{BH}_{4}\right)_{2}$. We optimize the atomic positions and lattice parameters of all compounds involved in possible formation and dehydrogenation reactions. Both the crystal structure and the electronic structure indicate that the bonding between $\mathrm{B}$ and $\mathrm{H}$ atoms is covalent and that the bonding between $\mathrm{Be}$ and has a covalent as well as an ionic contribution. The crystal structure and the electronic density of states give evidence for $\left[-\mathrm{BH}_{4}-\mathrm{Be}-\right]_{n}$ (helical) polymers.

The enthalpies of possible formation reactions are calculated including zero point energy corrections. The latter are obtained by calculating the phonon frequencies of all compounds involved in the reactions. Since not only hydrogen but also boron and beryllium are relatively light elements, the zero point energies are relatively large for these compounds. The enthalpy of formation of $\mathrm{Be}\left(\mathrm{BH}_{4}\right)_{2}$ from the elements is $\Delta E=-0.39 \mathrm{eV} / \mathrm{H}_{2}$ and $\Delta H_{0}=-0.14 \mathrm{eV} / \mathrm{H}_{2}$.

$\mathrm{Be}\left(\mathrm{BH}_{4}\right)_{2}$ differs from other boranates and alanates in that its dehydrogenation to the elements is thermodynamically slightly more favorable than dehydrogenation via the simple hydride $\mathrm{BeH}_{2}$. In alkali or alkaline earth boranate and alanate, dehydrogenations always occur via the alkali or alkaline earth simple hydride. The different behaviors of $\mathrm{Be}\left(\mathrm{BH}_{4}\right)_{2}$ are mainly caused by the high stability of bulk beryllium metal.

$\mathrm{Be}\left(\mathrm{BH}_{4}\right)_{2}$ follows the general trends in the formation energies that have been observed in alkali and alkaline earth alanates and boranates. Boranates are more stable than the corresponding alanates, lighter cations give compounds that are more unstable, and alkaline earth compounds are more unstable than alkali compounds. ${ }^{45}$ Indeed, $\mathrm{Be}\left(\mathrm{BH}_{4}\right)_{2}$ is less stable than $\mathrm{LiBH}_{4}$ or $\mathrm{Mg}\left(\mathrm{BH}_{4}\right)_{2}$. We have not come across a mention of beryllium alanate in the literature, which might indicate that this compound would be too unstable.

\section{ACKNOWLEDGMENTS}

The authors wish to thank R. A. de Groot for helpful discussions and J. J. Attema for the use of his imaging software. This work is part of the research programs of "Advanced Chemical Technologies for Sustainability (ACTS)" and the "Stichting voor Fundamenteel Onderzoek der Materie (FOM)," both financially supported by the "Nederlandse Organisatie voor Wetenschappelijk Onderzoek (NWO)."
${ }^{1}$ F. Schüth, B. Bogdanovíc, and M. Felderhoff, Chem. Commun. (Cambridge) 20, 2249 (2004).

${ }^{2}$ A. Züttel, Naturwiss. 91, 157 (2004).

${ }^{3}$ Y. Nakamori, K. Miwa, A. Ninomiya, H. W. Li, N. Ohba, S. I. Towata, A. Züttel, and S. I. Orimo, Phys. Rev. B 74, 045126 (2006).

${ }^{4}$ T. J. Frankcombe, G. J. Kroes, and A. Züttel, Chem. Phys. Lett. 405, 73 (2005).
${ }^{5}$ K. Miwa, N. Ohba, S. I. Towata, Y. Nakamori, and S. I. Orimo, Phys. Rev. B 69, 245120 (2004).

${ }^{6}$ Y. Wu and R. M. Mohring, Abstr. Pap. - Am. Chem. Soc. 226, U576 (2003).

${ }^{7}$ P. Vajeeston, P. Ravindran, A. Kjekshus, and H. Fjellvåg, J. Alloys Compd. 387, 97 (2005).

${ }^{8}$ M. Au, A. Jurgensen, and K. Zeigler, J. Phys. Chem. B 110, 26482 (2006). 
${ }^{9}$ J. H. Wee, K. Y. Lee, and S. H. Kim, Fuel Process. Technol. 87, 811 (2006).

${ }^{10} \mathrm{M}$. Au and A. Jurgensen, J. Phys. Chem. B 110, 7062 (2006).

${ }^{11}$ K. Miwa, N. Ohba, S. Towata, Y. Nakamori, and S. Orimo, J. Alloys Compd. 404-406, 140 (2005).

${ }^{12}$ Y. Nakamori, A. Ninomiya, G. Kitahara, M. Aoki, T. Noritake, K. Miwa, Y. Kojima, and S. Orimo, J. Power Sources 155, 447 (2006).

${ }^{13}$ K. Miwa, M. Aoki, T. Noritake, N. Ohba, Y. Nakamori, S. I. Towata, A. Züttel, and S. I. Orimo, Phys. Rev. B 74, 155122 (2006).

${ }^{14}$ Y. Nakamori, H. W. Li, K. Miwa, S. Towata, and S. Orimo, Mater. Trans. 47, 1898 (2006).

${ }^{15}$ P. Vajeeston, P. Ravindran, A. Kjekshus, and H. Fjellvåg, Appl. Phys. Lett. 89, 071906 (2006).

${ }^{16}$ X. B. Yu, D. M. Grant, and G. S. Walker, Chem. Commun. (Cambridge) 37, 3906 (2006).

${ }^{17}$ K. Chłopek, C. Frommen, A. Leon, O. Zabara, and M. Fichtner, J. Mater. Chem. 17, 3496 (2007).

${ }^{18}$ M. J. van Setten, G. A. de Wijs, and G. Brocks, J. Phys. Chem. C 111, 9592 (2007).

${ }^{19}$ Also referred to as beryllium borohydride in the older literature.

${ }^{20}$ The $\mathrm{Be}\left(\mathrm{BH}_{4}\right)_{2}$ itself, however, will probably never be used as a hydrogen storage material in large scale commercial applications because of the toxicity of beryllium gas.

${ }^{21}$ J. P. Perdew, J. A. Chevary, S. H. Vosko, K. A. Jackson, M. R. Pederson, D. J. Singh, and C. Fiolhais, Phys. Rev. B 46, 6671 (1992).

${ }^{22}$ G. Kresse and D. Joubert, Phys. Rev. B 59, 1758 (1999).

${ }^{23}$ P. E. Blöchl, Phys. Rev. B 50, 17953 (1994).

${ }^{24}$ G. Kresse and J. Furthmüller, Phys. Rev. B 54, 11169 (1996).

${ }^{25}$ G. Kresse and J. Furthmüller, Comput. Mater. Sci. 6, 15 (1996).

${ }^{26}$ G. Kresse and J. Hafner, Phys. Rev. B 47, 558 (1993).

${ }^{27}$ S. G. Louie, S. Froyen, and M. L. Cohen, Phys. Rev. B 26, 1738 (1982).

${ }^{28}$ P. E. Blöchl, O. Jepsen, and O. K. Andersen, Phys. Rev. B 49, 16223 (1994)

${ }^{29}$ Using potentials with less electrons that are kept frozen and varying smaller PAW cutoff radii.
${ }^{30}$ L. G. Hector, J. F. Herbst, W. Wolf, P. Saxe, and G. Kresse, Phys. Rev. B 76, 014121 (2007).

${ }^{31}$ F. D. Murnaghan, Proc. Natl. Acad. Sci. U.S.A. 30, 244 (1944).

${ }^{32}$ G. Kresse, J. Furthmüller, and J. Hafner, Europhys. Lett. 32, 729 (1995).

${ }^{33}$ M. J. van Setten, M. A. Uijttewaal, G. A. de Wijs, and R. A. de Groot, J. Am. Chem. Soc. 129, 2458 (2007).

${ }^{34}$ R. C. Weast and M. J. Astle, CRC Handbook of Chemistry and Physics, 62nd ed. (CRC, New York, 1982).

${ }^{35}$ H. I. Schlesinger, H. C. Brown, and E. K. Hyde, J. Am. Chem. Soc. 75, 209 (1953).

${ }^{36}$ D. S. Marynick and W. N. Lipscomb, Inorg. Chem. 11, 820 (1972).

${ }^{37}$ G. S. Smith, Q. C. Johnson, D. K. Smith, Jr., D. E. Cox, R. L. Snyder, R.-S. Zhou, and A. Zalkin, Solid State Commun. 67, 491 (1988).

${ }^{38}$ P. Vajeeston, P. Ravindran, A. Kjekshus, and H. Fjellvåg, Appl. Phys. Lett. 84, 34 (2004).

${ }^{39}$ E. S. Makarov and K. I. Tobelko, Dokl. Akad. Nauk SSSR 275, 91 (1984).

${ }^{40}$ A. Peles, J. A. Alford, Zhu Ma, Li Yang, and M. Y. Chou, Phys. Rev. B 70, 165105 (2004).

${ }^{41}$ A. Aguayo and D. J. Singh, Phys. Rev. B 69, 155103 (2004).

${ }^{42}$ P. Vajeeston, P. Ravindran, R. Vidya, H. Fjellvåg, and A. Kjekshus, Cryst. Growth Des. 4, 471 (2004).

${ }^{43}$ O. M. Løvvik, Phys. Rev. B 71, 144111 (2005).

${ }^{44}$ M. J. van Setten, G. A. de Wijs, V. A. Popa, and G. Brocks, Phys. Rev. B 72, 073107 (2005).

${ }^{45}$ M. J. van Setten, V. A. Popa, G. A. de Wijs, and G. Brocks, Phys. Rev. B 75, 035204 (2007).

${ }^{46}$ K. P. Huber and G. Herzberg, Molecular Spectra and Molecular Structure (Van Nostrand Reinhold, New York, 1979).

${ }^{47}$ H. Hemmes, A. Driessen, and R. Griessen, J. Phys. C 19, 3571 (1986).

${ }^{48}$ M. D. Senin, V. V. Akhachinskii, Y. E. Markushkin, N. A. Chirin, L. M. Kopytin, I. P. Mikhalenko, N. M. Ermolaev, and A. V. Zabrodin, Inorg. Mater. 29, 1416 (1993). 